\title{
Under the framework of "the silk road economic belt" Analysis and enlightenment of the integration of hospital and medical tourism industry in China
}

\author{
$\mathrm{Xu} \mathrm{Xu}$ and Nan $\mathrm{Wu}$
}

The First Affiliated Hospital of Xi'an Jiao Tong University Medical College, Xi'an, Shanxi, China

Keywords: the silk road economic belt, Chinese hospital, medical tourism, industry integration

\begin{abstract}
By reading amount of literatures, the paper explains and demonstrates situations and development modes of the medical tourism industry based on the "Silk Road Economic Belt", puts forward policy suggestions to integration of development of Chinese hospitals and medical tourism industry, the purpose of paper is to improve the economy of medical-tourism industry, spread the Chinese culture and carry forward the Chinese medical technology.
\end{abstract}

\section{“丝绸之路经济带” 框架下 中国医院与医疗旅游产业融合的分析及启示 徐旭 武楠 \\ 西安交通大学医学院第一附属医院，西安，陕西，中国}

关键词：丝绸之路经济带；中国医院；医疗旅游；产业融合

中文摘要. 文章通过阅读大量文献, 研究“丝绸之路经济带”框架下医疗旅游产业现状及发展模 式，对中国医院与医疗旅游产业融合发展提出相关政策建议，对提高医疗旅游产业经济、传 播中国文化、发扬中国医疗技术起到至关重要的作用。

\section{1. 医疗旅游的定义}

世界旅游组织在《旅游业 21 世纪议程》中，将医疗旅游(Medical tourism)定义为“以医疗 护理、疾病与健康、康复与修养为主体的旅游服务”, 具体含义可理解为人们由于常住地的医 疗服务不够完善或者太昂贵, 被异地(尤其是异国)价格低廉、具有特色的医疗、保健、旅游 等服务所吸引, 到异地接受医疗护理、疾病治疗、保健等医疗服务与度假、娱乐等旅游服务 的过程 ${ }^{[1]}$ 。

Perfetto 等研究者认为，医疗旅游应该只包括以择期手术为目的的旅行，不包括移民者在 他国进行医疗服务、计划外的紧急手术和门诊治疗 ${ }^{[2]}$ 。而 Melinda 等研究者 ${ }^{[3]}$ 则认为，尽管牙 科治疗不需要住院, 也应该算在医疗旅游的范围内。我国研究者 ${ }^{[4-5]}$ 则认为, 目前国际医疗旅 游需求可分为“治”为主和“疗”为主两大类。“治”为主的医疗旅游需求, 可分为基本无生命危险 并且医疗资源较为稀缺的项目、客源国尚未开发或被法律禁止的医疗项目(如坆胎、干细胞治 疗等)。以“疗”为主要目的医疗旅游需求, 主要为康复理疗类项目(如医疗检查、美容等)。

\section{2. 研究方法}


通过文献分析可以了解医疗旅游的研究现状、热点和不足，为确立研究目标和内容提供 了依据。在数据库 CNKI 中检索中文文献，应用检索式“旅游经济”，共检索文献 127 篇; “医 疗旅游”共检索文献 1814 篇; “丝绸之路经济带”共检索文献 6860 篇。

\section{3. 医疗旅游的成因及类型}

从客流方向看，早期的医疗旅游主要是发展中国家患者为了获得更好的医疗效果从而前 往发达国家就诊, 现在则表现为双向流动, 既有发展中国家的患者前往发达国家, 也有发达 国家的旅客前往发展中国家进行医疗旅游, 主要原因是减少等候治疗时间与相对低廉的成本。

从地理格局看，有的国家是医疗旅游的出口国(提供医疗旅游的国家)，有的为进口国(医 疗旅游旅客来源国), 有的同时兼具进口与出口业务。受社会、文化和语言因素的影响, 医疗 旅游目的地选择具有地域性特点。例如约 $70 \%$ 前往新加坡和马来西亚的患者来自东南亚地区 国家，前往古巴的医疗旅游患者主要来自拉丁美洲和中美洲地区，而约旦则吸引来自也门、 巴林、苏丹、沙特阿拉伯等国家和地区的患者，阿尔及利亚和利比亚的患者则会前往突尼斯 寻求医疗服务。有的国家因为具有较大的移民群体(如印度)或极具特色的医疗旅游项目(如泰 国)而吸引全球患者前往 ${ }^{[6]}$ 。而本文主要研究丝绸之路经济带框架下国际以及国内的医疗旅游 产业。

\section{4. 医疗旅游的特征}

医疗旅游是融合医学治疗与旅游观光的独特旅游方式, 它呈现出不同的特征，主要表现 为以下三点:

\section{1 反向流动特征}

在过去的二十年里，“反全球化”态势已经形成。为了医疗保健，发达国家的患者旅行到 欠发达国家，涉及成本、可接触的治疗等原因，揭示了保健领域的内隐观念 ${ }^{[7]}$ 。

Sammita 认为这批反向流向的患者，是为了规避他们自己国家的健康护理服务，一定程 度上它们是难以接触的、不受欢迎的、伴有负担过重的公共卫生系统以及长时间的等待 ${ }^{[8]}$ 。

\section{2 短距离流动特征}

欧洲、泰国、印度等国家的患者跨越国界, 到有类似的(或补充)设施和文化的邻近国家， 并且旅行成本最小化 ${ }^{[7]}$ 。John 认为医疗旅游不是乘坐航运从北方国家到南方国家, 它是相对 短距离的、区域的、交叉边界的、离散的(不跨越文化的界限)和有限的吸引力 ${ }^{[9]}$ 。

\section{3 移民返回特征}

许多医疗游客是海外移民，特别是美国的墨西哥人、印度和中东的移民。同样，第二代 海外移民，包括阿拉伯人和印度人，为了医疗护理迁移到“合适”的文化环境里 ${ }^{[7]}$ 。他们是为 了在熟悉的文化背景里寻求更便宜的医疗护理 ${ }^{[9]}$ 。

\section{5. 医疗旅游产品的主要类型} 产品 ${ }^{[10]}$ 。

卜克曼(Bookman)认为医疗旅游项目是将“高质量的医疗服务与旅游相结合”的医疗旅游

医疗旅游产品是旅游目的地为满足医疗旅游者的各类旅游需求，而提供的各种接待条件 和相关服务的总和。医疗旅游产品的类型、数量和质量决定了医疗旅游发展的水平。在医疗 旅游的目的地国中, 韩国是重要的代表国, 对医疗旅游的入境游客有较大的关注度, 国家医 疗旅游经验丰富, 医疗旅游产品综合性强、类型多样。韩国是近一二十年里, 医疗旅游发展 十分迅速的国家，尤其是在整形外科方面，已成为重要的医疗旅游目的国之一。 
根据医疗旅游者不同的医疗旅游目的, 可以将医疗旅游产品总结为以下几个不同的类型:

第一类, 治疗疾病类型的医疗旅游, 具体的医疗旅游产品有心脏类疾病的治疗, 肝脏、 肾脏等内科疾病的治疗，以及神经类疾病的治疗等等。

第二类，整容塑形类型的医疗旅游，旅游者为了整形美容进行医疗旅游，这是现代医疗 旅游的典型形式。目前, 韩国、马来西亚、泰国、新加坡等国家整形医疗旅游已经成为不可 忽视的吸引国外医疗旅游者的重要因素。

第三类, 养生康健类型的医疗旅游产品, 其是最为传统的医疗旅游产品形式, 温泉旅游 是最早的旅游形式, 近十几年来, 温泉的治疗养生的功能被不断地开发出来, 从医疗保健方 面来提高温泉旅游的发展水平，同时温泉旅游也是医疗旅游的重要产品形式。日本作为温泉 资源众多的国家之一, 其温泉医疗的发展程度较为成熟, 已形成以温泉为依托的健康医疗旅 游产品。其次, 随着人们越来越注意用药的健康性, 绿色药品开始受到欢迎, 中医药产品逐 渐被人们接受并重视, 韩国在植物药品的研制方面有很大的优势, 特别是在美容类的医药市 场上有举足轻重的地位。再次, 健康检查也是人们进行医疗旅游的重要动机之一, 在快节奏 的社会进程中，人们越来越多地注意到自己和周围亲人的健康状态，处于亚健康状态的人们 在旅游放松的过程中希望对自身的身体状况有所了解或者进行改善, 因此健康检查与休闲旅 游相结合也是医疗旅游的重要产品形式之一。

第四类，体验、观光、游览类型的医疗旅游产品，具体的医疗旅游活动主要有参加医药 博物馆，药用植物园，寻访特色名医等。

第五类，特殊目的的医疗旅游产品，比如早前中国大陆孕妇为了避免计划生育政策的控 制，到其他国家进行生产并获得当地永久居住权的活动 ${ }^{[11]}$ 。

\section{6. 医疗旅游的影响因素}

医疗旅游的影响因素包括促进因素和阻碍因素。医疗旅游发展的促进因素，一是医疗费 用和治疗等待时间的差异。这是促使很多国外病人前往发展中国家寻求医疗服务的主要因素 [12]。发达国家的高成本费用、较长的等待时间与发展中国家相对较低的成本费用、进行手术 无需等待的差异, 是吸引游客前往目的地国家进行医疗旅游的重要因素。比如 Kittikanya 研 究发现, 泰国的医疗费用仅仅是新加坡的 $1 / 2$, 美国的 $1 / 5^{[13]}$ 。参加医疗旅游的人愿意到目的 地享受质量更好、成本更低、无等待时间的目的地非紧急医疗服务 ${ }^{[14]}$ 。二是旅游目的地医疗 服务水平的提高和设施的完善。通过国际医疗机构认证已经成为招徕高质量国际医疗旅游者 的重要手段, 在这一方面许多发展中国家走在了前列。三是具有地方特色的治疗方式。比如 印度传统医学阿育吠陀和瑜伽、韩国整形美容、泰国丰胸和变性以及中国中医技术等吸引了 大量的医疗旅游者前往学习、医疗及度假。四是互联网通讯技术的兴起、健康保险公司的发 展和医疗服务机构的迅速发展。这些因素进一步推动了医疗旅游的发展。Lydia L. 等认为美国 居民参加医疗旅游主要受医疗保险政策的影响 ${ }^{[15]}$ 。当然医疗旅游的影响因素还包括国家政府 部门的推动、世界经济全球化进程的加快、航空运输业的迅速发展与国际旅行费用的降低等 等。学者们对医疗旅游发展的阻碍因素也进行了研究。学者们发现精湛先进的医疗技术的缺 乏、语言交流的障碍道德规范的约束和政治的不稳定等因素都制约着医疗旅游的发展 ${ }^{[16] 。}$

\section{7. 丝绸之路经济带构架下国际医疗旅游发展模式及分析}

\section{1 亚洲医疗旅游产业发展现状}

目前在国外，医疗旅游已经成为一个颇具规模的产业。医疗旅游不仅为那些需要保健旅 游的人带去了健康和快乐，也为提供医疗旅游服务的地区带来了很好的经济效益。

\section{2 印度}

印度成立了国家医疗旅游委员会和医疗旅游协会, 制定了一整套推行医疗旅游的法规、 
政策、战略与计划。印度通过一年一度的旅游博览会推广本国医疗旅游。民族传统医学可为 医疗旅游发展增加砝码, 印度的阿育吠陀医学和悉达医学就已吸引了部分欧美患者, 成为印 度发展医疗旅游的特色资源。据印度医务人员估计, 在英国做一个心脏手术平均费用是 3 万 英镑, 而孟买只需要 6000 英镑。据印度工业联合会的一份报告称, 印度的收费一般是欧美国 家的 $1 / 10$ 。另外, 别看印度大多数公立医院条件差, 平均每 1000 人分配不到一张病床, 但印 度大部分私立医院的条件不比欧美大城市医院差, 甚至还有所超越。医疗质量有保证是吸引 人的关键。埃斯科特医院去年完成 4200 例心脏手术, 死亡率只有 $0.8 \%$, 感染率只有 $0.3 \%$, 而在发达国家, 同样的手术平均死亡率达到 $1.2 \%$, 感染率则是 $1 \%$ 。会讲英语的医护人员也 是印度医疗旅游工程的重要法宝之一。印度大部分的医护人员都会讲英语, 这就为来自世界 各地的患者能够与他们沟通提供了方便。目前, 医疗旅游每年能为印度创造 4000 万个就业岗 位，同时医疗保健业也是印度经济中最大的服务业之一，有 400 多万从业人员。

\section{3 新加坡}

被世界卫生组织列为亚洲拥有最佳医疗系统国家的新加坡，近年成为周边国家富商喜欢 前来看病的地方, 许多印尼富商甚至每年定期到新加坡住院一个星期, 接受健康检查, 演变 成医疗度假的形态。新加坡也积极开拓医疗旅游, 2005 年共有 40 万海外患者来新加坡接受 包括 $\mathrm{X}$ 光检测, 眼、心脏、大脑和癌症等手术在内的一系列医学治疗带来了约合 38.8 亿元 人民币的收益。新加坡政府将生化产业列为国际发展计划, 促进医疗产业高质量发展, 力求 成为亚洲医学中心。新加坡政府极力推广本国的医疗旅游, 新加坡医疗旅游成功的关键是政 策支持和语言优势。在新加坡, 英语为其官方语言, 对于西方人士无沟通障碍; 汉语使用人 口占 $75 \%$, 有利于开发华人市场。不过, 新加坡的医疗旅游正面临马来西亚等国的挑战。为 开拓市场，新加坡将瞄准中国和印度的富人们。

\section{4 泰国}

泰国在建设亚洲保健观光中心政策支持下, 吸引着越来越多的外国人飞往泰国去享受阳 光、沙滩、庙宇等旅游服务以及专业、价格低廉的医疗服务。在泰国曼谷的 Bumrungrad 医院， 每年 100 万病人中, 近一半是外国病人, 其中最多来自美国的病人, 接着是来自阿联酋、孟 加拉国、英国、日本、澳洲、柬埔寨和缅甸。该医院还和泰国航空结盟, 提供便宜的配套服 务吸引外国人。泰国一些医院聘请的职员, 有讲流利英语的, 也有能翻译阿拉伯语、孟加拉 语、汉语、日语、韩语甚至西班牙语, 以加强其同新加坡和印度的竞争力。在泰国, 医院提 供的医疗服务包括牙科、激光、整形外科、丰胸、变性以及面部美容等。在泰国全球最有名 的变性手术医院之一的 Yanhee 医院, 隆胸手术费用只是美国的 1/10 至 1/5。泰国医疗旅游兴 起, 不仅仅是价格优势, 还有一个原因就是患者在泰国可以保护隐私。品质、匿名、便利等 因素是吸引外国人选择去泰国旅行治疗的主要原因。

\section{5 韩国}

韩国鉴于本地有医生成为干细胞疗法的权威, 希望能将韩国建设成为世界医疗保健旅游 的目的地, 让无法在所属国找到救药的外国病人前来就诊。一些国家的游客专门到韩国进行 干细胞或胚胎细胞的治疗, 这种治疗在一些国家还不能进行, 有些国家的法律也不允许这类 治疗。这类治疗给一些痽疾病人带来了新的希望。另外, 大多数外国患者到韩国寻求医疗服 务, 主要是牙医、整形外科和体格检查。另据报道, 由于越来越多的日本、中国等亚洲国家 女性为整容来到韩国, 韩国首尔也启动为外国整容游客联系整容外科医院的工作。韩国医疗 旅游协会表示将医疗旅游集中在整形美容上的这次尝试, 将比韩国政府正在推进的医疗旅游 事业取得更好的效果。

综合上述国家医疗旅游发展状况, 可看出医疗旅游产业发展的成功因素与政府的强力支 持、医疗资源服务定位明确、医疗推广宣传到位、医疗技术优良以及良好的医疗服务质量等 因素息息相关。 


\section{8. 中国医疗旅游的现状}

\section{1 理论研究现状}

最早介绍医疗旅游的是武强 ${ }^{[17]}$ ，他从科普角度介绍了当时新兴的一种以海洋资源为依托 的疗养方式; 王砚较早正式提出医疗旅游开发的思想 ${ }^{[18]}$; 杨鸿尔介绍了埃及以其城市和矿泉、 硫磺泉水以及干燥性气候等自然旅游资源开展医疗旅游, 吸引了一批批慕名而来的治疗皮肤 病、关节炎等疾病的旅游者 ${ }^{[19]}$; 李芳、李耀中针对四川乐山旅游饭店经营问题提出要看到医 疗旅游的需求 ${ }^{[20]}$; 任圆圆 ${ }^{[21]}$ 和徐菲、陈婉丽[22]等学者从我国医学、医疗角度和国外医学、医 疗角度, 对开发医疗旅游项目的做法和部分经验进行了介绍和说明; 李佳、苑粀频首次对我 国医疗旅游业的发展做了分析和研究, 对照国外医疗旅游发展好的国家和地区, 分析了我国 开展医疗旅游的现状、问题, 并提出对策和展望 ${ }^{[23]}$ 。从 2007 年开始, 有关医疗旅游的研究就 更加关注我们地区、地域文化和各区域资源的禀赋情况。如杨晓霞等从喀斯特洞穴的自然研 究角度探讨开展洞穴医疗旅游 ${ }^{[24]}$; 孙永平、刘丹对中医药旅游的概念进行了界定和探讨, 并 通过对中医药旅游资源的分析, 提出了发展中医药旅游的建议 ${ }^{[25]}$; 田广增对医疗保健专项旅

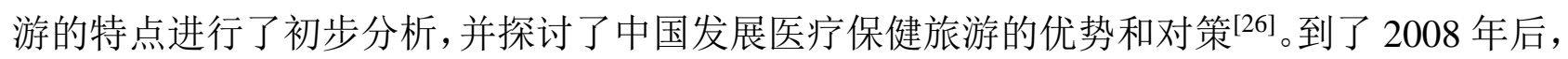
有学者开始从旅游产业模式和产业融合角度研究和关注医疗旅游, 如杨颖认为医疗旅游是旅 游产业演化、融合的高级表现形式 ${ }^{[27]}$ 王琼、温小霓分析了西安医疗旅游的特点、发展空间, 并提出发展策略 ${ }^{[28]}$ 。

\section{2 产业发展现状}

从国际医疗旅游产业的发展来看, 全球目前已有 100 国家和地区正在开展医疗旅游的服 务项目, 而瑞士、韩国、泰国、印度等国家的医疗旅游产业发展尤为蓬勃。有统计显示, 全 球医疗旅游的游客数量已经从 2006 年的 2000 万人次, 增加到 2012 年的 4000 万人次, 还有 数据预测医疗旅游的年产值已经到达 1000 亿美元的规模, 年增速在 $20 \%$ 左右 ${ }^{[28]}$ 。从国内医 疗旅游产业的发展来看, 我国医疗旅游还处于起步阶段, 在我国内地, 北京、上海、广州等 少数一线城市对于发展医疗旅游产业表现出极大的热情, 积极学习国际医疗发达国家的经验, 部分也着手制定本地区医疗旅游产业发展的相关政策和措施。同时, 部分旅游城市, 如三亚、 杭州、北戴河等也相继推出医疗旅游服务。当然, 我国香港与台湾地区医疗旅游的发展水平 明显高于内地, 但总体而言, 医疗旅游对于我国仍属一个较新的领域, 可能还只是一个新概 念或理念, 在产业发展的规模和意义上还存在一定的局限性, 最多也就是保健而已, 不能成 为真正意义上的医疗旅游, 因此我国的医疗旅游还处于起步阶段, 最多也是初步成长期。确 切来说，医疗旅游产业还没有形成。

\section{9. 我国医疗旅游开发管理存在的问题}

尽管我国已经开始关注和发展医疗旅游, 但由于前期产业发展的基础薄弱, 目前我国医 疗旅游开发管理仍存在以下几方面的问题和现象。

\section{1 发展级别较低}

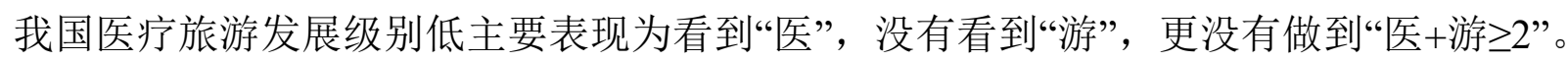
看到“医”而未看到“游”表现为, 在医疗时, 旅游不应出现和伴生; 而旅游时, 医疗应当退出 和忽略。此类状况也是医疗和旅游的两极状态。没有做到“医+游 $\geq 2$ ”的表现为我国有些地区的 医疗旅游已初有成效, 如香港、台湾、澳门等地区, 但是大部门地区和城市, 没有形成气候, 主要是没有将医疗业和旅游业很好地融合, 两个产业链是平行的、分离的。没有突破以“治”

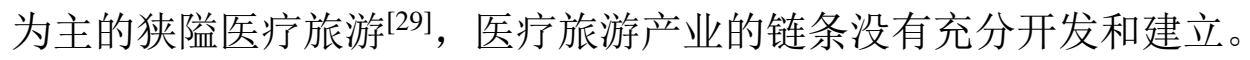

\section{2 资源整合不足}

我国医疗旅游资源整合不足，主要表现在医疗资源整合不足，旅游资源整合不足和医疗 
旅游资源融合不足几个方面。医疗资源整合不足表现为对医疗资源中的现代医疗技术和产品、 传统医疗手段以及产品的开发和利用不足; 旅游资源整合不足主要变现为对相关旅游资源的 开发和利用不足。一方面, 在自然旅游资源的使用上, 一般以园区等形式为主, 没有进一步 开发和升级; 另一方面, 对于人文旅游资源的开发和利用, 没有形成产业规模和优势, 医疗 资源容和不足, 主要是医疗与旅游两个行业契合度不够。两者各自关注各自传统领域, 在契 合的条件、基础和保障方面整合不足。

\section{3 品牌建设滞后}

纵观全球医疗旅游发达的国家和地区, 特色品牌十分突出。新加坡用全世界最佳的医疗 系统为旅游者提供精密的医疗服务; 韩国设立整容美容支援中心, 提供美容特色医疗旅游项 目; 高质量医疗服务和手术成功率是印度医疗的一大特色 ${ }^{[30]}$ 。我国拥有悠久的医药历史和灿 烂的医药文化, 但作为国际医疗旅游来说, 这一点似乎并不太突出, 而且明显落后于亚洲其 他国家。

\section{4 管理盲区较多}

新兴产业多以融合姿态出现, 涉及部门行业和领域较为广泛。其管理主体和客体较为复 杂, 医疗旅游更为复杂。其所含医疗和旅游这两大行业本身非常敏感, 将二者融合起来管理 就更复杂且盲区也较多。

\section{5 市场需要开发不充分}

市场需求开发不充分的主要表现是对于医疗旅游只停留在“医”而不在“游”。医疗旅游产 业链并没有开发充分, 没有从医疗角度充分开发旅游项目, 也没有从旅游角度充分开发医疗 项目。大多数医疗旅游的发展只是在病重时治病, 在旅游时休闲, 两者融合的产业需求没有 充分契合 ${ }^{[31]}$ 。

\section{0. 对中国发展医疗旅游的启示}

\section{1 构建医疗旅游发展模式}

我国具有医疗服务成本低、旅游资源丰富、医疗技术水平较高等众多优势，但与印度、 泰国、马来西亚、韩国等周边国家和地区相比, 我国医疗旅游业仍处于起步阶段。因此, 在 对医疗旅游业发达国家的产业模式和成功经验进行研究和分析的基础上，应结合我国的实际 情况, 构建适合我国医疗旅游业的发展模式, 并研究具体的发展战略; 以特定地区为对象, 研究独具地方特色的个案医疗旅游实践模式; 探讨如何聚焦重点医疗项目, 利用我国传统的 中医药优势，开发特色医疗旅游产品，打造中西结合的国际医疗旅游品牌。

\section{2 提供国际化的医疗服务}

医疗旅游地的医疗服务质量是影响医疗旅游者决策的重要因素，医疗服务的国际认证程 度和医疗服务形象的好坏是影响医疗旅游者进行选择的重要标准, 但目前我国医疗卫生机构 在申请国际认证方面还不够重视, 国内仅有 20 余家医疗卫生机构通过 JCAHO 认证。因此, 相关医疗卫生机构应当研究并建立一套与国际标准接轨的诊疗服务流程和医疗技术标准; 重 视声誉和服务质量, 建立国际化的医疗设施和服务质量管理体系; 提高管理水平, 制定符合 国际标准的医疗机构管理标准，以吸引国内外医疗旅游者。

\section{3 完善医疗旅游政策法规}

目前, 我国医疗旅游业还缺乏专门的法律法规, 行业监管机制也有待完善, 许多缺乏相 关资质的机构或个人涉足医疗旅游业, 这势必会扰乱医疗旅游业的健康发展。因此政府应进 一步加强行业监管, 建立相应的准入和退出机制, 完善医疗旅游业的配套法律法规, 促进医 疗旅游业健康有序发展此外, 由于我国规定公立医院提供特需服务的比例不能超过全部医疗 
服务的 $10 \%$ ，并限制公立医院广告营销。这就导致了掌握我国大部分医疗资源的公立医院难 以开展相关的医疗旅游业务。同时, 由于我国对社会办医的限制以及传统医疗体制的束缚, 我国现有的民营医疗机构在服务规模和医疗水平方面竞争力不足。因此, 对于现存的不利于 我国医疗旅游业发展的政策法规，还需要政府部门和相关学者进一步的研究和探讨。

\section{4 培养和吸引医疗旅游人才}

目前, 我国医疗旅游领域的复合型人才十分缺乏，尚未建立相应的人才培养体系。因此， 下一步应就医疗旅游领域专业人才所需的核心能力和专业技能、人才培养体系、人才培养方 案与课程设置进行探讨和研究。此外, 针对医疗旅游业相关的医院、健康管理公司、蛇行摄、 酒店、保险公司等机构的相关人员, 如何根据不同机构工作人员原有的工作特点和专业技能, 系统性的设置相应的培训课程和培训计划, 挖掘现有人才资源的潜力, 使之能够较快适应国 内以及国际医疗旅游业发展的需要, 也是亟待研究的问题之一。同时, 由于我国高端医疗人 才的缺乏，对于医生多点执业的政策细节尚不明朗，限制了高端医疗人才的有效利用，使得 我国医疗旅游业的发展缺乏核心资源。因此，在重视人才培养、逐步实现医生多点执业的基 础上，应当通过政策优惠等方式吸引国外优秀的专业人才，来提高我国医疗旅游业的服务水 平和竞争力 ${ }^{[32]}$ 。

\section{References}

[1] Hung-chi Chen, Hsin-chih Kuo, Kuo-piao Chung, et al. Classification and Comparison of Niche Services for Developing Strategy of Medical Tourism in Asian Countries, International Surgery, vol. 95(2), pp. 108-116, 2010.

[2] Perfetto, Dholakia, Exploring the Cultural Contradictions of Medical Tourism, Consumption Markets \& Culture, vol. 13, pp. 399-417, 2010.

[3] Melinda M, Ethical and Medical Dilemmas of Space Tourism, Advances in Space Research, vol. 37, pp. 1823-1827, 2006.

[4] Justin M, Schneider, Medical Tourism in the Backcountry: Alternative Health and Healing in the Arkansas Ozarks, Signs, vol. 36, pp. 419-326, 2011.

[5] Liu Tingfang, Jiao Yahui, Dong Siping, Li Dachuan, Xue Haining, Zhang Dan, Experience and its reference on international medical tourism industry, Chinese Hospitals, vol.20, pp.1-6, 2016.

[6] Chengjuan Liao, The development, influence and enlightenment of the international trade in health services, Health Economics Research, vol. 5, pp. 56-59, 2016.

[7] John Connell, Contemporary medical tourism: conceptualization, culture and commodification, Tourism Management, vol. 34, pp. 1-13, 2013.

[8] Sammita Jadhav, Rajiv Yeravdekar, Meenal Kulkarni, Cross - border Healthcare Access in South Asian Countries: Learnings for Sustainable Healthcare Tourism in India, Procedia - Social and Behavioral Sciences, vol. 157, pp. 109-117, 2014.

[9] John Connell, From medical tourism to transnational health care? An epilogue for the future, Social Science \& Medicine, vol. 124, pp. 398-401, 2015.

[10] Bookman M, Bookman K, Medical tourism in developing countries, Basingstoke: Palgrav Macmillan, pp.6-12, 2007.

[11] Ben HaoBin Ye, Hanqin Zhang Qiu, Peter P Yuen, Motivations and experiences of Mainland Chinese medical tourists in Hong Kong, Tourism Management, vol. 32, pp. 1125-1127, 2011.

[12] Dehuang Ning, Juan Liu, Review on the development of International Medical Tourism, Journal of Central South University of Forestry \& Technology(Social Sciences), vol. 2, pp.1-4, 2013.

[13] Keckley PH. Coughlin S, Survey of Health Care Consumers Global Report: Strategic Implications, Washington: Deloitte Center for Health Solutions, 2011.

[14] Thomas C. Chuang, John S. Liu, Louis Y. Y. Lu, Yachi Lee, The main paths of medical tourism: From transplantation to beautification, Tourism Management, vol. 45, pp. 49-58, 2014.

[15] Lydia L. Gan, James R. Frederick, Medical Tourism: Consumers' Concerns Over Risk and 
Social Challenges, Journal of Travel \& Tourism Marketing, vol. 32, pp. 503 - 517, 2015.

[16] Yuqin Song, Degen Wang, A Comparison of medical tourism between domestic and overseas researched in the past decade, Geography and Geo-information Science, vol. 6, pp.105-110, 2011.

[17] Qiang Wu, Magical ocean therapy, Ocean world, vol. 10, pp.15, 1995.

[18] Yan Wang, Tourism: a promising industry in the West, Ethnic Unity, vol. 4, pp.61, 2000.

[19] Hongxi Yang, Egypt Tourism, China National Conditions and Strength, vol. 7, pp. 56-57, 2001.

[20] Fang Li, Yaozhong Li, How to enhance the inner construction of Leshan tourist hotel? Resource Development \& Market, vol. 2, pp.13-14, 2002.

[21] Yuanyuan Ren, Patients of Europe and the United States are attracted by medical tourism in Asia, Medicine Healthcare Apparatus, vol. 12, pp. 29-30, 2005.

[22] Fei Xu, Wanli Chen, India's medical tourism industry development and its enlightenment to our country, South Asian Studies Quarterly, vol. 4, pp.118-121, 2006.

[23] Jia Li, Tongtong Yuan, The analysis of medical tourism development in our country, Economic vision, vol.9, pp. 14-16, 2006.

[24] Xiaoxia Yang, Xu Xiang, Daoxian Yuan, Summary on karst cave tourism research, Carsologica Sinica, vol. 26, pp.369-375, 2007.

[25] Yongping Sun, Dan Liu, The research on Chinese medical tourism, Market Modernization, vol. 5, pp.357, 2007.

[26] Guangzeng Tian, Medical Service Traveling Development Research of Our Country, Journal of Anyang Normal University, vol. 5, pp.93-96, 2007.

[27] Ying Yang, Industry Convergence: A New Perspective of Tourism Development Trends, Tourism Science, vol. 22, pp.6-10, 2008.

[28] Qiong Wang, Xiaoni Wen, Medical Tourism: The new Developmental Pattern of Tourism Industry in Xi'an, Journal of Xidian University (Social Sciences Edition), vol.5, pp.18-23, 2009.

[29] Yongli Liu, Yanling Huang, The development strategy research of China's medical tourism, Jiangsu Commercial Forum, vol.10, pp. 60-64, 2013.

[30] Pian Luo, India's medical tourism development analysis and its enlightenment to our country, Tourism overview, vol.1, pp. 63, 2014.

[31] Wei Yan, The present situation and the promotion strategy of medical tourism development in China, Research On Development, vol. 2, pp.153-15, 2015.

[32] Zhijie Wu, Qing Guo, International research status of medical tourism and its implications for China, Chinese Journal of Health Policy, vol. 11, pp.59-63, 2014. 